${ }^{28}$ Bookstein, J. J., et al., Fournal of the American Medical Association, 1972, 221, 368 .

29 Varady, P. D., and Maxwell, M. H., Fournal of the American Medical Association, 1972, 221, 365.

so Reiss, M. D., Bookstein, J. J., and Bleifer, K. M., fournal of the American Medical Association, 1972, 221, 374.

31 Goldman, A. G., Varady, P. D., and Franklin, S. S., fournal of the American Medical Association, 1972, 221, 378.

32 Baker, G. P., Page, L. B., and Leadbetter, G. W., New England fournal of Medicine, 1962, $267,1325$.

33 Hunt, J. C., Strong, C. G., Sheps, S. G., and Bernatz, P. E., American Fournal of Cardiology, 1969, 23, 434.

st Foster, J. H., et al., Surgery, 1966, 60, 240

35 Kaplan, N. M., Clinical Hypertension, pp. 203-242. New York, Medcom Press, 1973.

\section{Rifampicin or Ethambutol in the Routine Treatment of Tuberculosis}

Rifampicin and ethambutol have now been used for some years in the retreatment of tuberculous patients with tubercle bacilli resistant to the standard drugs. Their proved efficacy, their excellent toleration by patients, and their relative lack of toxicity have raised the question whether they might be given in primary treatment of newly diagnosed cases, especially as a substitute for para-amino salicylic acid (PAS), the most troublesome of the three classical drugs. A trial by the Medical Research Council was designed to answer this question. A secondary objective was to compare as follow-up treatment streptomycin plus high-dosage isoniazid, given under supervision twice weekly, with the classical PAS plus isoniazid, and with ethambutol or rifampicin plus isoniazid. The trial was co-operative and carried out in 51 centres in Britain. A report on it has recently been published. ${ }^{1}$

Patients with newly diagnosed pulmonary tuberculosis were allotted at random to one of four regimens, consisting of an intensive phase of three drugs daily for the first three months followed by two drugs as continuation therapy up to 12 months. The regimens were as follows: (1) Streptomycin $0.75 \mathrm{~g}$ plus isoniazid $300 \mathrm{mg}$ plus sodium PAS $12 \mathrm{~g}$, the PAS and isoniazid being given once daily or divided into two doses; this was followed by isoniazid plus PAS as continuation therapy. (2) Ethambutol $15 \mathrm{mg} / \mathrm{kg}$ was substituted for PAS and given with the isoniazid in a single dose daily; otherwise the regimen was as in (1). (3) Rifampicin $600 \mathrm{mg}$ was substituted for PAS and given with isoniazid in a single dose daily. (4) Treatment for the first three months was as in (1) but continuation treatment was with streptomycin $1 \mathrm{~g}$ twice weekly given under supervision with isoniazid $15 \mathrm{mg} / \mathrm{kg}$ plus pyridoxine $10 \mathrm{mg}$.

A total of 481 patients were admitted to the trial. Some were excluded from the analysis for various reasons, including primary drug resistance and early death (15 patients); 412 were left for analysis. The most important analysis was of the speed of conversion of the sputum to negative culture. This was significantly more rapid in the rifampicin group, and smearpositive culture-negative sputa were in the early months significantly more common. After two months culture was negative in $60 \%$ of the rifampicin group compared with $36-38 \%$ in the other three groups. At three months culture was negative in $81 \%$ compared with $49-66 \%$ in the other groups. At 12 months there was little difference between the groups, the figure being $\mathbf{9 8 \%}$ for the rifampicin group compared with $95-98 \%$ for the others. Though a few patients had single or a very few colonies in cultures at 12 months, these were all sensitive to streptomycin and isoniazid and on the basis of previous experience were not regarded as failures. Only four patients were judged to be failures, none in the rifampicin group. Two were in the PAS group and there was evidence from urine testing of irregularity in medication. The other two were unexplained.

Radiological comparisons were also made. Considerable improvement occurred in all groups, and there was little difference between them at 12 months.

The comparison of side effects and toxicity was clearly important. In the first three months side effects occurred in $21 \%$ of both the ethambutol and rifampicin groups compared with 30 and $32 \%$ in the two groups on PAS. A major alteration of treatment was necessary in $13 \%$ of the rifampicin, $17 \%$ of the ethambutol, and 20 and $27 \%$ of the PAS groups. Most of these were vestibular upsets attributed to streptomycin and hypersensitivity reactions, mainly attributed to streptomycin or PAS. Only one of the latter was attributed to ethambutol and none to rifampicin. "Gastric" side effects occurred in 4\% of the rifampicin, $1 \%$ of the ethambutol, and 9 and $11 \%$ of the PAS groups. Jaundice occurred in only 1 rifampicin patient but in 3 in the PAS groups. "Hepatitis" without jaundice was recorded in 4 rifampicin patients though in none of the other groups, but a subsidiary intensive study of 23 rifampicin and 31 PAS patients showed no difference in the incidence of hepatocellular dysfunction. As regards visual disturbance, a known hazard of ethambutol, minor abnormalities of fields were found in 6 of 99 ethambutol patients compared with 3 of 89 PAS ; only 1 (ethambutol) patient complained of blurring of vision. Ethambutol was stopped in several of these patients. "Blind" review of the tests by an independent ophthalmologist suggested that a presymptomatic toxic optic neuropathy had been present in 3 ethambutol and 1 PAS patient.

One can conclude from these investigations that rifampicin and ethambutol are both effective and relatively non-toxic substitutes for PAS, and the speed of sputum conversion will be enhanced by rifampicin. Liver toxicity with rifampicin does not seem to be a major problem. It is wise to carry out liver function tests at the start of treatment but to repeat them only if there is any symptomatic suggestion of hepatitis. There is still a slight worry about ophthalmological complications of ethambutol, though with this low dose the risk is clearly small. It is justifiable to carry out initial ophthalmological testing and to warn the patient to report at once if he develops dimness of vision, as subjective changes may precede objective. It is doubtful whether routine testing is necessary during treatment, especially if the low dose of $15 \mathrm{mg} / \mathrm{kg}$ is used.

It is now clear that the clinician can give rifampicin or ethambutol instead of PAS, and probably instead of streptomycin. He might do so as a routine, though there is still a slight concern about eye complications with ethambutol, which may occur late in treatment. But cost should also be considered. At present hospital prices $10 \mathrm{~g}$ of sodium PAS costs $2.5 \mathrm{p}, 1 \mathrm{~g}$ of ethambutol $22 \mathrm{p}$, and $600 \mathrm{mg}$ of rifampicin $68 \mathrm{p}$. The new drugs are therefore expensive. Clearly they are justified if there is any tolerance to standard drugs, but it may be argued that they should not be given as a routine until the price decreases. They are, of course, essential for patients with resistance to the classical drugs. The price also makes their use prohibitive in developing countries, certainly for routine treatment. There is perhaps a case for making them available for the treatment of patients with bacilli resistant to the standard drugs but if possible restricting their use to selected centres where they will be properly given. There is a grave risk that their widespread use by the less sophisticated will result in adding resistance to these drugs to the large and shameful pool of resistance to classical therapy.

1 British Medical Research Council Co-operative Trial, Tubercle, 1973, 54, 99. 